\title{
Multiparameter monitoring of short-term earthquake precursors and its physical basis. Implementation in the Kamchatka region
}

\author{
Sergey Pulinets ${ }^{1,}$, Dimitar Ouzounov ${ }^{2}$, Dmitry Davydenko ${ }^{1}$ and Alexei Petrukhin ${ }^{3}$ \\ ${ }^{1}$ Space Research Institute, RAS, 84/32 Profsoyuznaya str., 117997, Moscow, Russia \\ ${ }^{2}$ CEESMO, Chapman University, 1 University Drive, Orange, CA 92866, USA \\ ${ }^{3}$ Schmidt Institute of Physics of the Earth, RAS, Bolshaya Gruzinskaya, 10, 123242, Moscow, Russia
}

\begin{abstract}
We apply experimental approach of the multiparameter monitoring of short-term earthquake precursors which reliability was confirmed by the Lithosphere-Atmosphere-Ionosphere Coupling (LAIC) model created recently [1]. A key element of the model is the process of Ion induced Nucleation (IIN) and formation of cluster ions occurring as a result of the ionization of near surface air layer by radon emanating from the Earth's crust within the earthquake preparation zone. This process is similar to the formation of droplet's embryos for cloud formation under action of galactic cosmic rays. The consequence of this process is the generation of a number of precursors that can be divided into two groups: a) thermal and meteorological, and b) electromagnetic and ionospheric. We demonstrate elements of prospective monitoring of some strong earthquakes in Kamchatka region and statistical results for the Chemical potential correction parameter for more than 10 years of observations for earthquakes with $\mathrm{M} \geq 6$. As some experimental attempt, the data of Kamchatka volcanoes monitoring will be demonstrated.
\end{abstract}

\section{Introduction}

We consider the final stage of the earthquake preparation cycle as the process of approaching the open complexed system to a critical state, and atmosphere (and its ionized upper shell-ionosphere) are the integrated parts of this system. With this approach, it can be argued that the crustal deformation, foreshock activity, various geophysical anomalies recorded by ground-based monitoring methods, atmospheric and ionospheric anomalies registered from space are not independent processes. They are the parts of synergetic interaction of geospheres, and by their distribution in space, by the sequence of appearance in time allow to reveal what is called in synergetics "arrow of time" characterizing the irreversibility of the process. In the case when a core set of key parameters is available (as it was after the analysis of numerous publications on the earthquake in Aquila April 6, 2009) such directionality can be demonstrated experimentally (see Fig. 2 in [1]). However, the organization of operational forecasting by monitoring the large number of the necessary

* Corresponding author: pulse@rssi.ru 
data is not easy task, and new approaches should be investigated. We offer the use of socalled integral parameters characteristic to a synergistic approach. The advantage of these options is their availability (remote access), global accessibility (most used parameters - a remote sensing data from satellites) and reliability (according to our experience, their abnormal behavior seen in almost $100 \%$ of cases of strong $M>6$ earthquakes). These parameters are called integral because they characterize the state of the environment as a whole and reflect a significant change in its properties.

Our additional and very important task is to unite the probabilistic and deterministic approaches to the earthquake forecast what will prepare ground to physicists and seismologists to work together on this noble task.

\section{Integral parameters}

To demonstrate the rightness of our approach among large set of the physical precursors of earthquakes we selected two: one - the $b$ - value from the Gutenberg-Richter FrequencyMagnitude Relationship (FMR), and second - the correction of atmospheric chemical potential (ACP) which indicated the deviation of the latent heat transform in the boundary layer of atmosphere in the presence of ionization source. The first parameter is used in probabilistic approach by seismologists, and the second one - in deterministic approach exploring the physical short-term precursors.

\section{$2.1 b$-value as indicator of precursory period}

To characterise the regional seismicity Gutenberg and Richter [2] proposed the empirical relationship in the form:

$$
\log N=a-b M
$$

where $N-$ is a cumulative number of earthquakes with the magnitude higher than $M, a-$ coefficient characterising the regional seismicity, and $b$ - the parameter that seems characterises the state of the crust on different stages of the earthquake cycle. Usually, its value is closed to 1 , but on different studies of the seismic cycle it varies essentially and these variations look like indicator of the main shock approaching. At least in [3] the authors claim that "lower than average $b$-values characterize locked patches of faults (asperities), from which future mainshocks are more likely to be generated". In [4] the $b$ value drop was established as one of the three parameters which are used to identify the foreshock activity. This parameter attracted our attention because the $b$-value drop coincides in time with the period of increased radon emanation. In the left panels of the Fig. 1 the illustrations from the papers [5] and [6] are combined. Upper part of the left panel from top to bottom presents the seismic activity around the time of M6.9 Kobe earthquake which took place on 17 January 1995 in Japan, $b$-value, and fractal dimension of the seismic flux approximately equal to $2 b$. The lowest graph in the left panel shows the radon concentration in water [6]. The arrow shows the time coincidence of the parameters shown in the upper and lower graphs (to demonstrated radon variations more in details). As one can see, the sharp increase of radon emanation coincides in time with the period of $b$-value drop. However, the period of the sharp increase of radon concentration one can observe the gradual increase during several months that coincides with the period of increased $b$-value. 

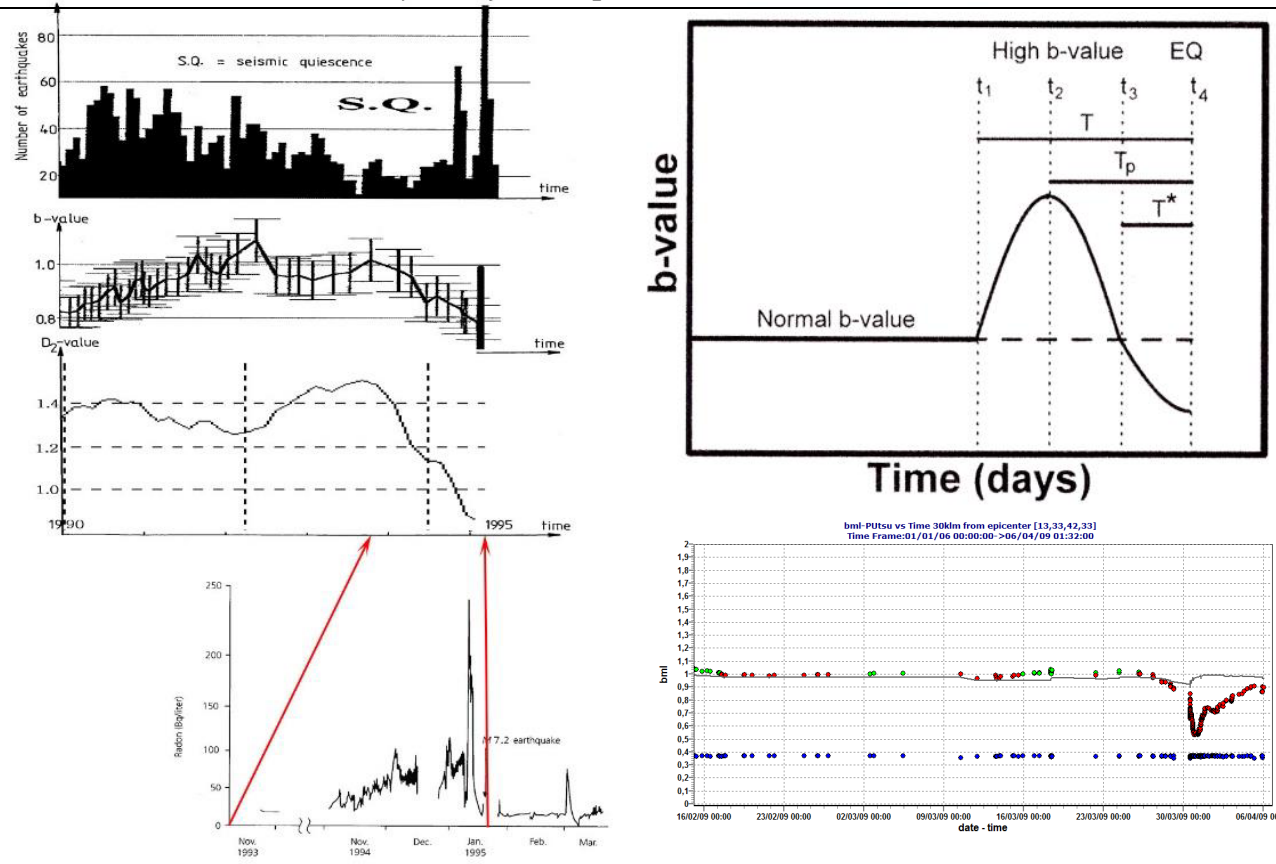

Time (days)

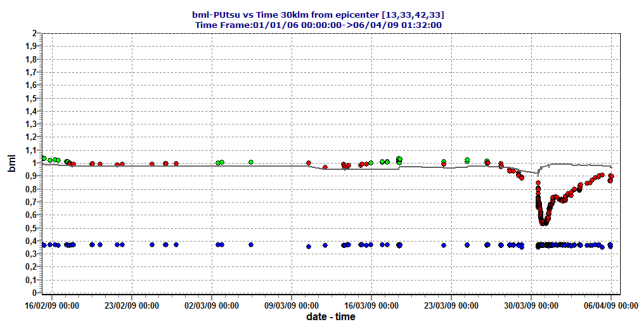

Fig. 1. Left panel from top to bottom: seismic activity around the time of Kobe earthquake, $b$-value variations, fractal dimension of the seismic activity variations after [5]), concentration of radon in water (after [6]; right panel, upper graph - schematic presentation of $b$-value anomalous variation before the main shock (after [7]), lower graph $-b$-value variations around the time of L'Aquila earthquake (after [4])

In review paper [7] the authors try to determine the precursory period with the help of $b$ value variations and determine the following phases of $b$-value anomaly before earthquake. This period denoted by $T$ is divided by 3 phases: $b$-value increase (from $t_{1}$ to $t_{2}$ ), $b$-value decrease from absolute maximum to the normal value (from $t_{2}$ to $t_{3}$ ), and $b$-value decrease from the normal value to the anomalous minimal values (from $t_{3}$ to $t_{4}$ ). The period from $t_{2}$ to $t_{4}$ when continuous decrease of $b$-value observed, called the precursory period $T_{P}$. Unlike the paper [7], Papadopoulos [4] considers only the $b$-value drop lower than normal, and in his graph we can see that minimum of $b$-value is reached before the main shock. Nevertheless, we can observe the slight increase of $b$-value on his graph (right left corner of the Fig. 1). Our comparison with the time scale of anomalous variations of $b$-value and physical precursors for L'Aquila earthquake [1], show the coincidence with Papadopoulos results.

The integral character of $b$-value precursors is determined by two factors: a) it is calculated not in a single point but integrates the seismic activity within some area around the epicenter; b) in one parameter are reflected the complex processes of the earth's crust transformation from elastic deformation up to brittle changes. Decrease of $b$-value is equivalent to decrease of fractal dimension what can be interpreted as consolidation and clustering of seismic activity (observed experimentally) and increase of cracks formation. Just these new cracks are the opportunity for radon to find the new ways to the ground surface what leads (together with stress storing equivalent the pressure increase) to the amplification of the radon flux before earthquakes.

However, it is not so simple to monitor the radon activity over the large areas of earthquake preparation zone, spatially with remote sensing from satellites. Recently we have reached possibility to get the radon proxy using the secondary thermal effects in atmosphere produced by air ionization. 


\subsection{ACP as a proxy of radon variations}

Due to its decay, radon is emitting the aparticles with energy $5.46 \mathrm{MeV}$. Taking into account the low ionization potential of atmospheric gases (10-20 eV), every aparticle emitted by radon can create in average $3 \cdot 10^{5}$ electron-ion pairs. The presence of ions in atmosphere creates possibility to the molecules of water vapor to join to these ions through the hydration process, which is different from condensation. In the process of condensation the chemical potential is equal to the Latent heat which is $Q=40.683 \mathrm{~kJ} / \mathrm{mol}$ or $U_{0}=0.422 \mathrm{eV}$ per one molecule. The process of evaporation/condensation been the phase transition of the first order always takes place during the chemical potential equality. However, new formed ions due to air ionization by radon have the chemical potential different from the water molecules. Therefore, in the one-component approximation we introduce the correction to the chemical potential $\Delta U$, which takes into account this difference, and the chemical potential in condition of external impact due to ionization can be expressed as:

$$
U(t)=U_{0}+\Delta U \cdot \cos ^{2} t
$$

where $U_{0}$ is the chemical potential for pure water, and $U(t)$ is the chemical potential where the ionization and hydration is taken into account. By $\cos ^{2} t$ we take into account the daily changes of the solar radiation.

Then we can express the air relative humidity in the following form:

$$
H(t)=\frac{\exp (-U(t) / k \cdot T)}{\exp \left(-U_{0} / k \cdot T\right)}=\exp \left(\frac{U_{0}-U(t)}{k \cdot T}\right)=\exp \left(-\frac{0.032 \cdot \Delta U \cdot \cos ^{2} t}{(k \cdot T)^{2}}\right)
$$

where $k$ - Boltzman constant, and T - air temperature. The increase of the water molecules chemical potential $\Delta U$ (which can be derived from (3)) indicates the strength of nucleation process and can be used as indicator of radon activity because the nucleation is proportional to the new ions' concentration which in turn is proportional to radon activity.

No one model of atmosphere takes into account the ionization impact, therefore the variations of the air humidity and temperature resulting from the radon activity will be interpreted by models as anomalous.

The experimental check made evident that the temporal morphology of $\Delta U$ (denoted by us as ACP) is very similar to radon behavior before earthquakes, and this parameter can be used to monitor activity from space.
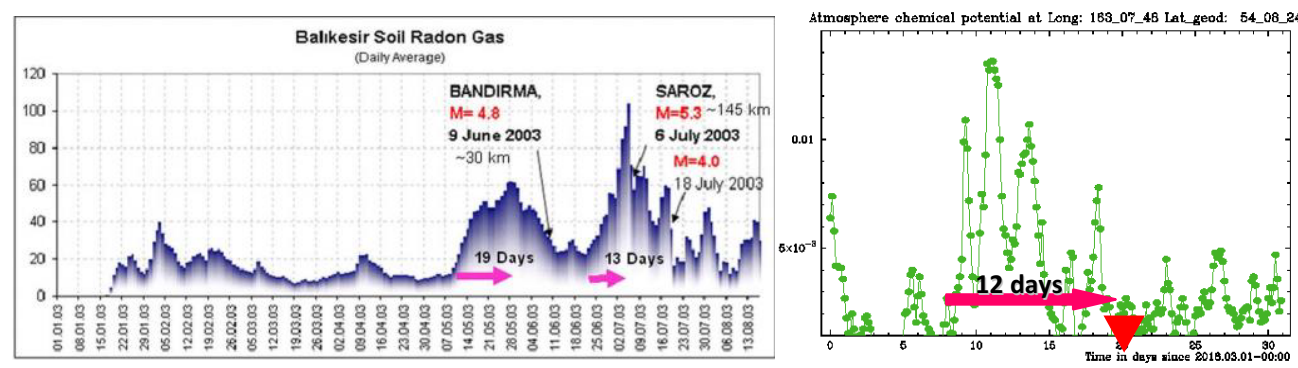

Fig. 2. Left panel - typical radon variations around the time of series of earthquake in Turkey; right panel ACP variations around the time of M6.7 earthquake on 20 March 2016 at Kamchatka 
As one can see from the Fig. 2 the ACP variations practically are the same as radon variations, even the temporal scale (time from start of parameter increase to the mainshock) is identical.

\section{Real time short-term precursors monitoring of Kamchatka}

Multiparameter monitoring permits to increase the reliability of earthquake forecast from the point of view of the forecast parameters determination. Selection of precursors is limited by their availability for different regions of the globe. In this regard, the satellite monitoring has advantage before the ground-based measurements. For Kamchatka region the three most reliable precursors were selected: OLR (Outgoing Longwave infrared Radiation), measured at the level of top of atmosphere (10-15 km of altitude), ACP derived from the satellite measurements of the air temperature and humidity at the altitude $100 \mathrm{~m}$, and GPS TEC anomalies. One can find in [1] their physical explanation and interrelation, here we want to demonstrate how the main forecast parameters are extracted from the measurements.

One of the advantages of mutiparameter monitoring is the different leading time to mainshock moment. The OLR appears the first and serves as the warning to start the realtime detailed monitoring of all parameters. Then the ACP parameter is used which has it main maximum within the time interval 18-4 days before the mainshock. And as the last usually appears the ionospheric anomaly which could be monitored both by GPS TEC or ground based vertical sounding monitoring. It is important to note that we are able using the satellite technologies to monitor not only temporal behaviour of precursory anomaly but also its spatial distribution. The left panel demonstrates the spatial distribution of OLR anomaly registered 01 of March 201619 days before the M6.4 earthquake on 20 March 2016 also mentioned in comments to the Fig. 2. The anomaly determines the region of the future earthquake epicenter position, while anomaly intensity permits to determine the earthquake magnitude. The right panel of the Fig. 3 shows the spatial distribution of ACP for the same earthquake registered 10 days before the earthquake. Taking into account that ACP repeats not only temporal but also the spatial behaviour of radon, from the size of anomaly we also can estimate earthquake magnitude using the Dobrovolsky formula for radius of earthquake preparation zone.
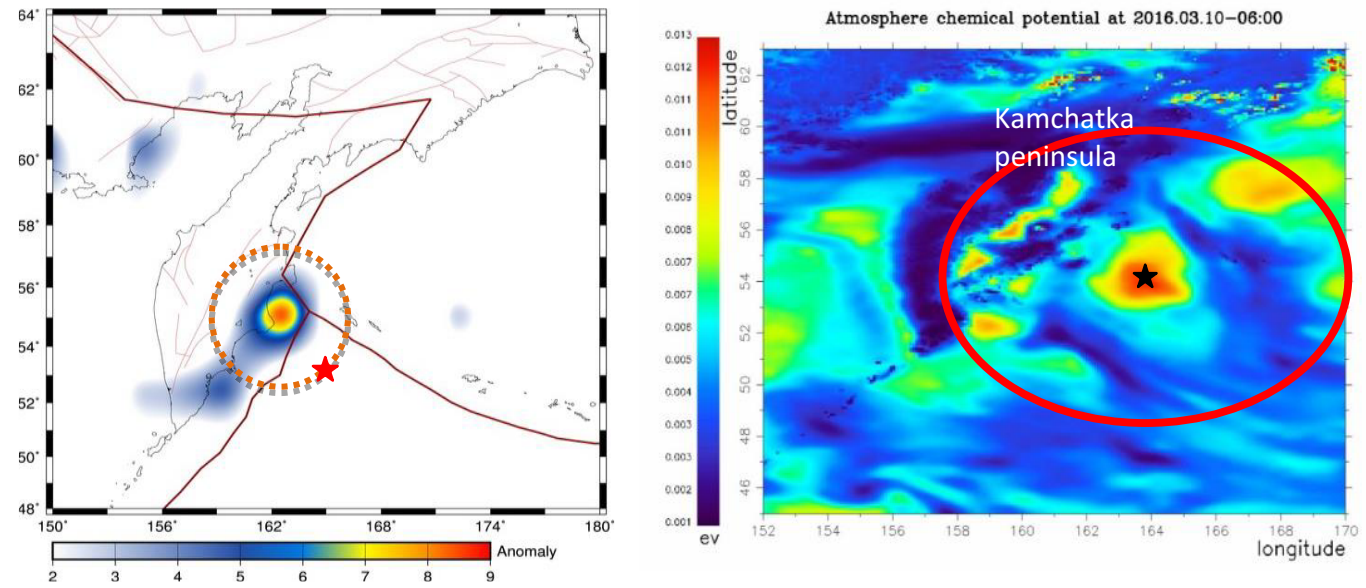

Fig. 3. Left panel - OLR anomaly registered on 01 of March 2016; right panel - ACP anomaly registered 10 of March 2016. Asterisks-epicenter position, red circle-Dobrovolsky zone 


\subsection{Statistical analysis of ACP variations at Kamchatka region}

We analyzed variations of ACP for 64 earthquakes at Kamchatka region during more than 11 years (from 2005 till part of 2016) and revealed self-similarity of the ACP precursors through the whole territory including Kuril islands and ocean around Kamchatka. The manifestation of precursors in ocean as it is seen at the Fig 3 confirms the gaseous (radon) source of the anomaly.

The results of this analysis are demonstrated in the Fig. 4 in the form of distribution of the leading time of main maximum in time series of ACP and minimum just before the earthquake. One can see that the distribution of maxima (red) has two peaks (-11 and -4$)$ before earthquake, while distribution for minima (blue) has major peak at the earthquake day and smaller one 2 days before earthquake

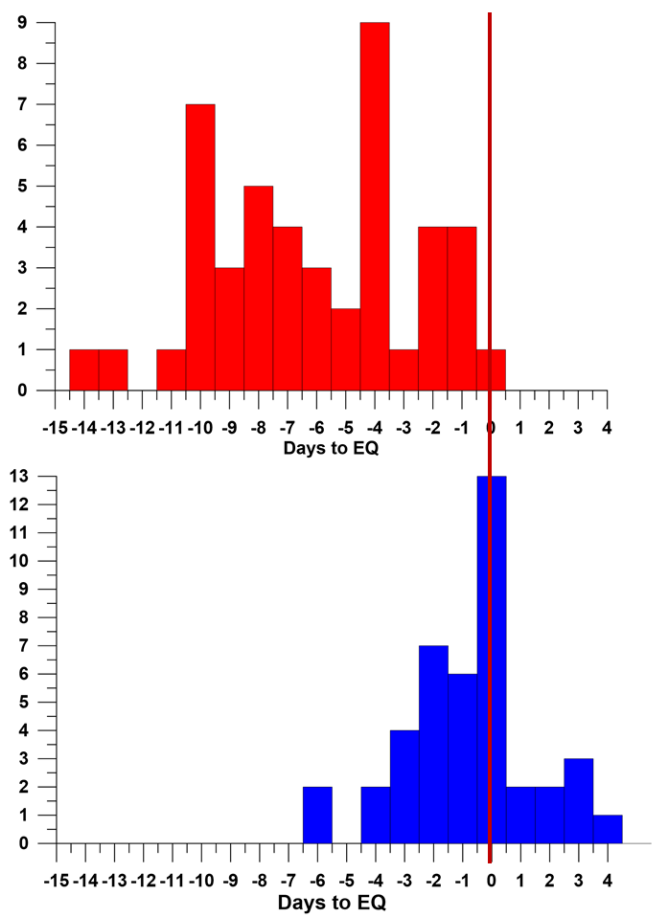

Fig. 4. Upper panel - distribution of leading time of the main maxima of the ACP time series; lower panel - distribution of leading time of the main miniima of the ACP time series. Vertical red line indicates the day of earthquake.

\section{2 lonospheric monitoring of Kamchatka region}

Unfortunately we had limited access to the data of Kamchatka GPS receivers network. Practically the data of only two receivers of IGS network were available (pets and yssk) what permitted us to provide the so called cross-correlation analysis of the ionospheric anomalies before earthquake at Kamchatka [8]. Advantage of this technique is in its imminent forecast ability: the drop of the cross-correlation coefficient is observed usually one day before the main shock. In the Fig. 5 is shown the example of real time monitoring of the GPS TEC for period of June-July 2012 when the series of $M \geq 6$ earthquake took place in the region. The effectiveness of the technology is obvious. 


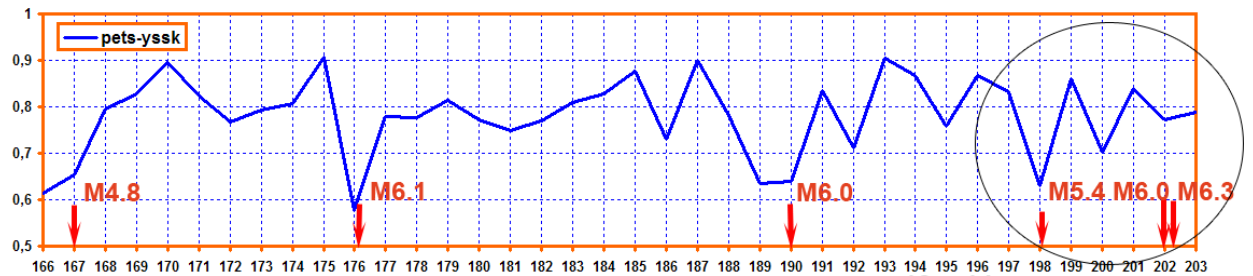

Fig. 5. Cross-correlation coefficient calculated for period June-July 2012 for GPS receivers pets and yssk.

\section{Volcanoes monitoring by ACP technique}

Existing reports on the radon activity during the volcanoes eruption [9] inspired us to check how the ACP variations correspond to the reported radon variations during volcano eruption. The results of comparison appeared amazing: the complete coincidence of radon and ACP variations was revealed. In the left panel of Fig. 6 the radon variations during Taal volcano (Philippines) eruption in October1994 are shown. In the right panel the Klyuchevskoi volcano activity in ACP for period from 1 August til 16 September 2016 is shown.
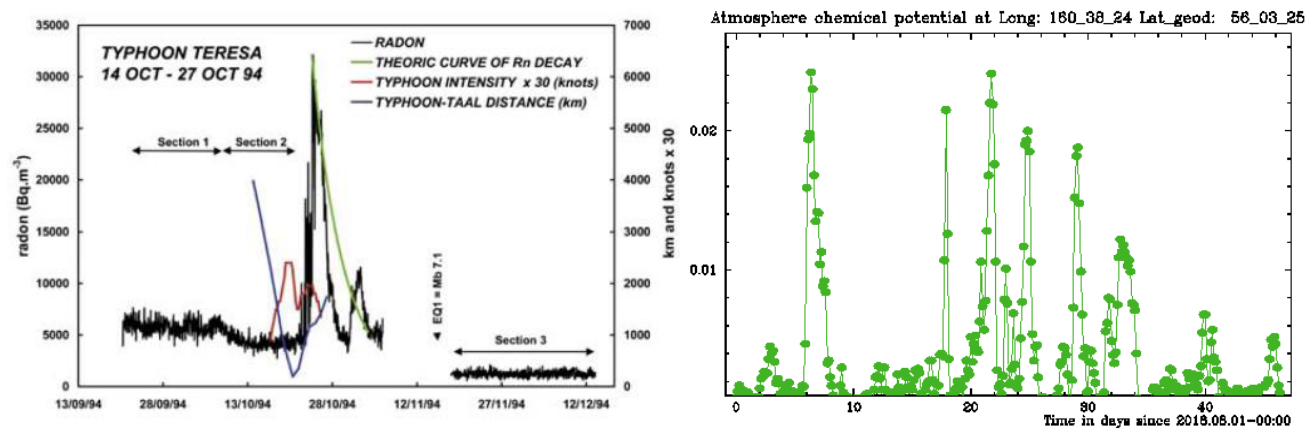

Fig. 6. Left panel - radon activity (black line) and radon decay curve (green line) are shown during Taal volcan eruption on October 1994; right panel - variations of ACP during Klyuchevskoi volcano activity in August-September 2016.

\section{References}

1. S.A. Pulinets, D.P. Ouzounov, A.V. Karelin, D.V. Davydenko, Geomagn. Aeron., 55, 540 (2015)

2. B. Gutenberg, C.F. Richter, Bull. Seism. Soc. Am., 34, 185 (1944)

3. D. Schorlemmer, S. Wiemer, M. Wyss, JGR, 109, B12307, (2004)

4. G.A. Papadopoulos, M. Charalampakis, A. Fokaefs, G. Minadakis, Nat. Haz. Earth Syst. Sci., 10, 19 (2010)

5. B. Enescu, K. Itu, Annuals of Disast. Prev. Res. Inst., Kyoto Univ., No.46 B (2003)

6. G. Igarashi, S. Saeki, N. Takahata, and K. Sumikawa, Science, 269, 60 (2010)

7. J.-H. Wang, K.-C. Chen, P.-L. Leu, C.-H. Chang, J. Seismology, 20, 905, (2016)

8. S.A. Pulinets, T.B. Gaivoronska, A. Leyva Contreras, L. Ciraolo, , Nat. Haz. Earth Syst. Sci., 4, 697 (2004)

9. P. Richon, J.-C. Sabroux, M. Halbwachs, et al., GRL, 30, 1481 (2003) 